\title{
The Gut Microbiota in Epilepsy: Current Concepts of Mechanisms and Potential Therapeutics
}

\author{
Rikky Dwiyanto Sulistyo
}

\section{ABSTRACT}

\begin{abstract}
Epilepsy is a non-communicable brain disorder characterized by an individual's proclivity for spontaneous epileptic seizures. Epilepsy may be classified into six types: genetic, structural, metabolic, infectious, immunerelated, and unexplained causes. Numerous current findings have shown evidence that an imbalance in the gut microbiota is a cause of epilepsy. Between the gut microbiota and the brain systems, there are five putative communication pathways. The neuroendocrine hypothalamic-pituitaryadrenal (HPA) axis, intestinal bacteria's production of neurotransmitters, the intestinal immune system, and the relationship between the intestinal mucosal barrier and the blood-brain barrier are among them. Future epilepsy interventions might include modifications of antiepileptic medications, a ketogenic diet, and probiotics as a possible treatment in the gut flora. However, further research is required to assess long-term therapeutic benefits.
\end{abstract}

Keywords: Epilepsy, gut microbiota, nketogenic diet, eurological disease, probiotics.

\section{INTRODUCTION}

Epilepsy is a non-communicable brain disorder characterized by an individual's proclivity for spontaneous epileptic seizures (Fisher et al., 2014). It affects people of all ages, with an estimated global burden of 45.9 million patients and 126,055 epilepsy-related mortality each year (Global Burden of Disease Study, 2016). Epilepsy, which affects around 46 million people globally, accounts for a significant portion of the global disease burden, according to the 2016 Global Burden of Illness Collaborators (Global Burden of Disease Study, 2016). Cerebral abnormalities, central nervous system (CNS) infection, trauma, stroke, and genetic changes in epilepsy genes are all major causes of epilepsy. According to current research, the gut microbiota plays an essential role in epilepsy pathogenesis (Scheffer et al., 2017). The microbiome-gut-brain axis is gaining popularity as a new area for understanding the complexities of many neurological illnesses. Recent research in the area of epilepsy have proposed that the gut microbiota may contribute to the maintenance of an inflammatory state that may promote drugresistant seizures (Borghi \& Vignoli, 2019; Cryan et al., 2020). In this review, we examine the existing data for gut microbiota disturbances in epilepsy, as well as prospective therapies for the microbiota in modulating epilepsy risk as seizure interventions.

\section{EPILEPSY}

Epilepsy is a chronic neurological condition defined by at least two unprovoked seizures separated by more than 24 hours, one unprovoked (or reflex) seizure with a recurrence probability of more than $60 \%$ in the following 10 years, or a proven epilepsy syndrome (Fisher et al., 2014). Genetic, structural, metabolic, infectious, immune-associated, and unknown are the six etiology groups of Epilepsy (Global Burden of Disease Study, 2016). The gut microbiota has been related to a variety of neurological diseases, however there is little evidence regarding in this area. As a result, identifying the involvement will pique researchers' interest in conducting in-depth study into the gut-brain axis' role in epilepsy manifestation (Borghi \& Vignoli, 2019).

\section{GUT MicrobIOTA}

The intestinal (or gut) microbiota is a population of bacteria found in the gastrointestinal system. It is a subset of the larger microbiota, but it is unquestionably the most diverse and essential (Yamashiro, 2017). It weighs roughly $1.5 \mathrm{~kg}$ and is made up of at least 500 distinct bacteria species categorized into 45 genera and 14 families. Firmicutes (including Lactobacillus), Bacteroidetes, Actinobacteria, and Proteobacteria (including Escherichia) are the four most common bacterial phyla in the human digestive system (Rinninella, et al., 2019). Bacteroides species on its own account for about $30 \%$ of all intestinal bacteria, implying that this genus is particularly significant in the host's functioning (Rinninella, et al., 2019).

Some species are extremely beneficial, such as Bacteroides thetaiotaomicron, which greatly improves the body's carbohydrate metabolism, while others can be hazardous, such as Clostridium difficile, whose action is usually constricted by the presence of other microbial species but can cause diarrhea and fever in some cases (Zocco et al., 2007). The great majority of "good" gut bacteria is secreting mucus to protect the host (humans), which functions as a barrier 
between germs and the cells that make up the intestinal mucosa (Jakobsson et al., 2015). It also boosts the inflammatory response and immunological defenses against pathogens from the outside (Mendes et al., 2019). For these reasons, the microbiota has caught the interest of medical researchers across the board, because, unlike certain variables that cannot be changed and influence the disease development (such as age and genetics), the microbiota composition can be altered, at least theoretically. The gutbrain axis in neurological illnesses is thought to be a mechanism capable of impacting brain functions favorably or adversely through the activity of the gut microbiota.

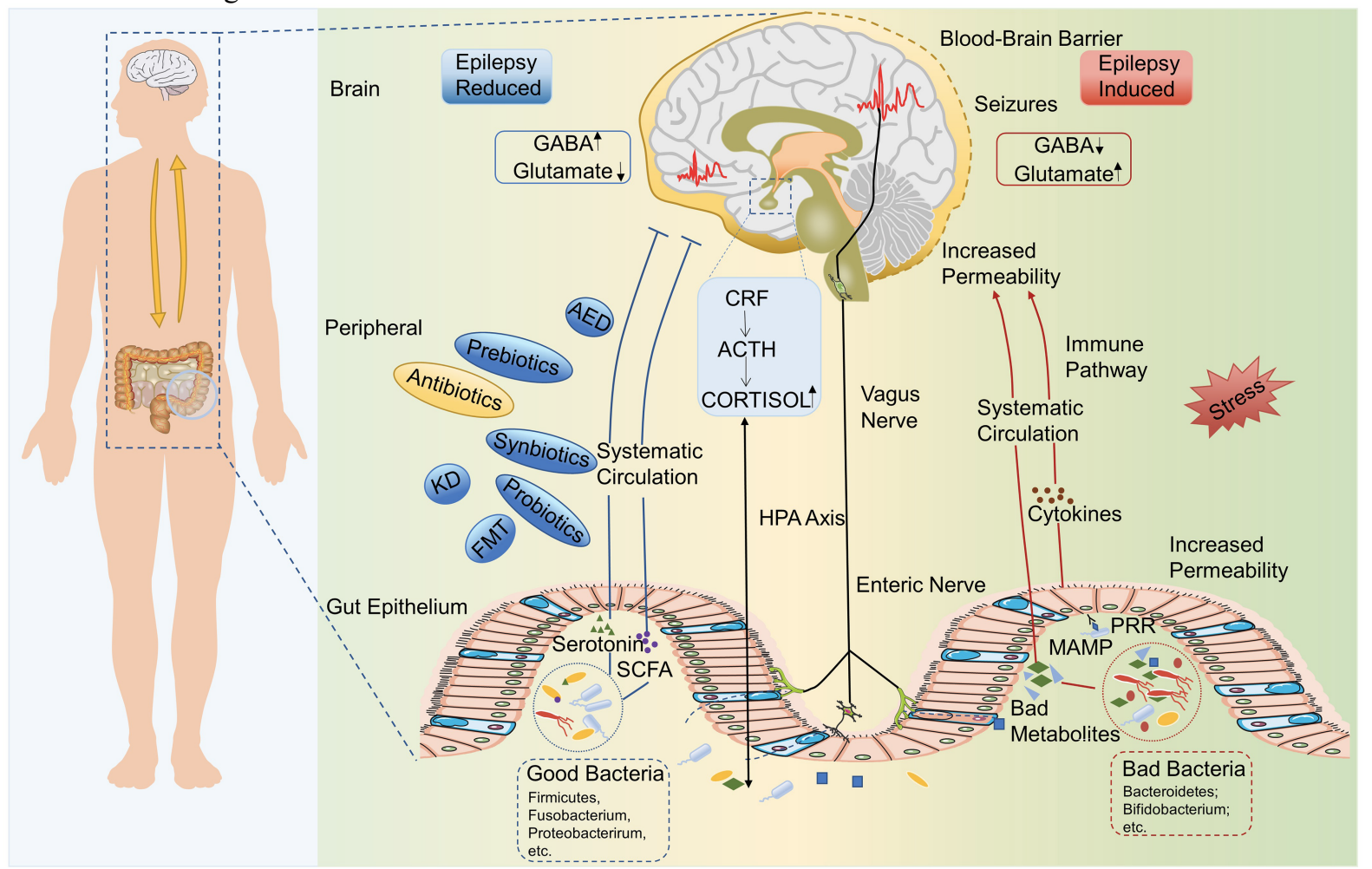

Fig. 1. The microbiome-gut-brain axis in epilepsy pathogenesis. Changes in the gut microbiota may increase the synthesis of epilepsy-inducing compounds and the release of inflammatory substances, leading to an aberrant GABA/glutamate ratio and, ultimately, epilepsy. In a healthy gut microbiota, beneficial metabolites like SCFAs and serotonin are produced, which may help to prevent epilepsy. The connection between the gut microbiota and epilepsy is also influenced by the HPA axis, enteric nervous system, and vagus nerve system.

Adrenocorticotropic hormone (ACTH); Antiepileptic drug (AED); Corticotrophin-releasing factor (CRF); Hypothalamic-pituitary-adrenal (HPA); gaminobutyric acid (GABA); Ketogenic diet (KD); Microbe-associated molecular pattern (MAMP); Short-chain fatty acid (SCFA); Pattern recognition receptor (PRR).

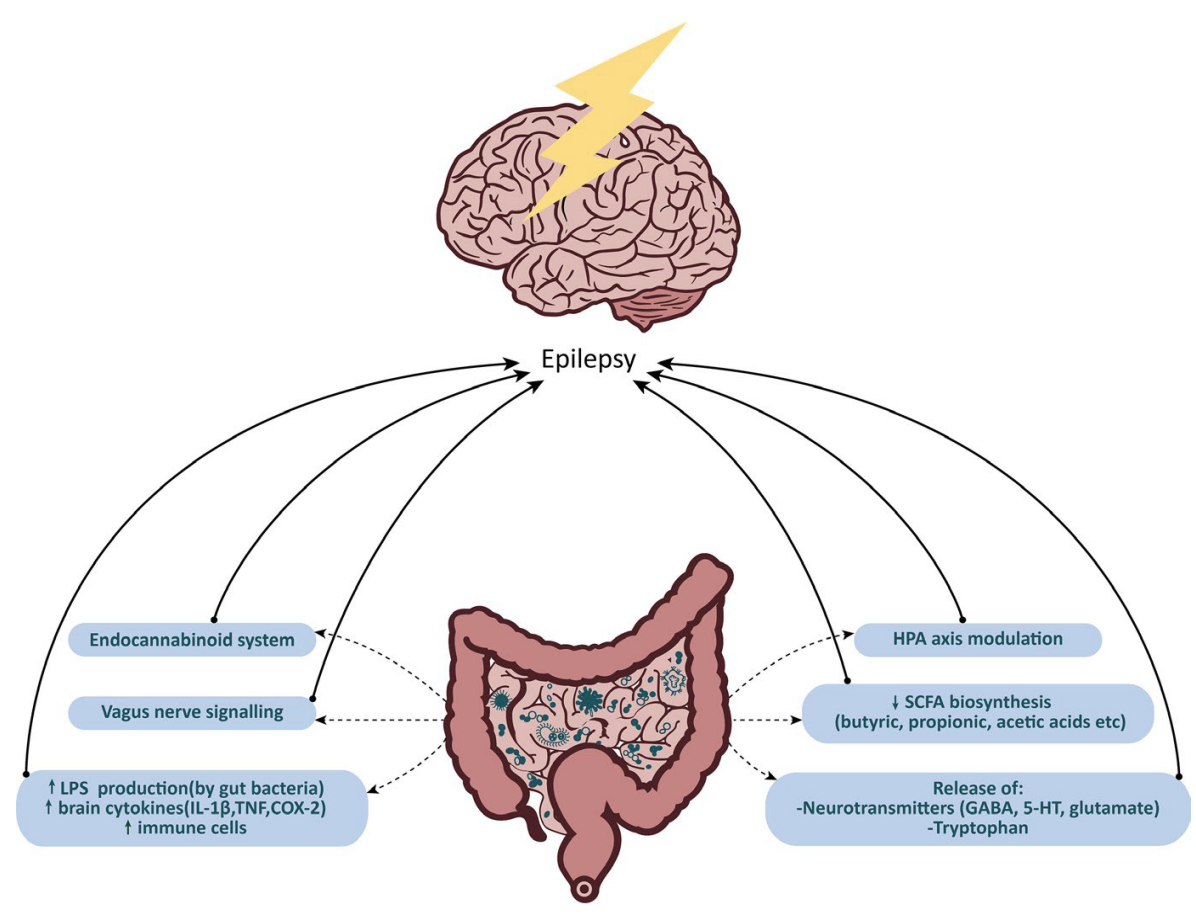

Gut Dysbiosis

Fig. 2. Inflammatory factors and another pathway induced epilepsy. 


\section{Gut Microbiota In EPILEPSy}

Various studies have found evidence that an imbalance in the gut microbiota is a cause of epilepsy, and hence a refractory illness. Between the gut microbiota and the brain systems, there are five putative communication pathways; neural connections, the neuroendocrine hypothalamicpituitary-adrenal (HPA) axis, intestinal bacteria production of neurotransmitters, the intestinal immune system, and finally, the interaction between the gut mucosal barrier and the bloodbrain barrier (Wang \& Wang., 2016; Mayer et al., 2015). In the meantime, various pathophysiological pathways are now being studied, including the possibility of a relationship between gut dysbiosis and epileptogenesis (Erny et al., 2015; Warner, 2019). The first pathway involves the release of neurotransmitters (particularly serotonin [5-HT], aminobutyric acid [GABA], and glutamate) or their precursors (tryptophan and its metabolites) by gut microbes, which has a direct influence on brain networks (O'Mahony et al., 2015). This has an impact on the CNS's balance of activation and inhibition (see Fig. 1). The second one is the neural signaling (Warner, 2019). Gut microbiota activate afferent neurons of the enteric nervous system (ENS) after vagus nerve stimulation promotes anti-inflammatory activity under homeostatic settings. A disruption in normal bacterial population or function might throw this balance off, leading to an increase in inflammation (Blander et al., 2017). Furthermore, the gut microbiota decline in normal biosynthesis of short-chain fatty acids (SCFA) (such as butyric, propionic, and acetic acids) that have been shown to have anti-inflammatory properties is a highly effective pathogenetic mechanism for stimulating secondary neuroinflammation that can lead to seizure (van de Wouw et al., 2018) (see Fig.1). Changes in the gut microbiota (composition or functional profile) may also alter seizure processes by disturbing the HPA axis and normal stress response, or by modifying the amounts of brain-derived neurotrophic factor (BDNF). Gut dysbiosis may also disrupt the endocannabinoid system (Al-Ghezzi et al., 2019), which may be associated to epilepsy development.

Therefore, seizures and epileptogenesis may be caused by a "leak" in the barrier system's integrity (intestinal mucosal barrier and blood-brain barrier), which is known to be influenced by the gut microbiota. Changes in the intestinal microbiota profile may result in an overabundance of lipopolysaccharide (LPS)-producing microbes, which causes greater intestinal immunological barrier permeability (due to elevated LPS levels) (Blander et al., 2017; Balosso et al., 2014). Theoretically, increased expression of brain cytokines such as interleukin (IL-1), tumor necrosis factor (TNF), and cyclooxygenase (COX-2) mediates the action of lipopolysaccharide on seizure development (Maroso et al., 2010). As a result, peripherally generated inflammatory factors may increase the permeability of the blood-brain barrier, allowing pro-inflammatory mediators to enter the CNS (see Fig. 2). Overall, different microbiota gut brain axis pathways appear to be engaged in the etiology and maintenance of epileptic activity. Gut dysbiosis should be restored as a potential moderator of these abnormalities. Antiepileptic medications, dietary changes, and probiotic supplementation are among those now being studied, since they might represent future therapeutic methods for treating epilepsy (Blander et al., 2017; Balosso et al., 2014; Maroso et al., 2010).

\section{Potential Epilepsy Treatment Using Microbiota TARGETS}

\section{A. Antiepileptic Drugs in Gut Microbiota}

Various drug-metabolizing enzymes in the gut microbiota alter their pharmacology, resulting in interindividual variability in drug effectiveness and toxicity (Zimmermann et al., 2019). Clonazepam, for example, is an anticonvulsant and anti-anxiety medicine that the gut bacteria reduce and metabolizes, leading to drug toxicity (Raymond et al., 2016). Non-antibiotic medications have an effect on the gut microbiota. In big research that looked at the impact of 1,197 non-antibiotic medications on the gut microbiota of 40 people, 24 percent of the pharmaceuticals with human targets reduced the development of one strain in vitro (Maier et al., 2018). Antiepileptic medications such as carbamazepine, valproic acid, and lamotrigine alter the constitution of the gut microbiota (Sgritta et al., 2019). Valproic acid administration during pregnancy in mice leads to a change in fecal microbiota (Drekonja et al., 2015), with more Firmicutes and fewer Bacteroidetes, which might be related to autism spectrum disorders-like behavior in children (Drekonja et al., 2015; de Theije et al., 2014).

There is some literature that suggests the gut microbiota may have a role in epilepsy. A 22-year-old patient with Crohn's disease and a 17-year history of seizures underwent a fecal microbiota transplant (FMT) to treat Crohn's disease, according to $\mathrm{He}$ et al., (2017) Despite of terminating antiepileptic medication therapy with sodium valproate, the patient was found to be seizure-free after 20 months. Apart from that, Stokes et al., (2014) discovered that lamotrigine may reduce $E$. coli growth by suppressing bacterial ribosome biogenesis. More study about the interaction between antiepileptic medications and gut microbes will aid in the development of novel antiepileptic therapies based on the gut microbiota regulatory concept. Antiepileptic medications' metabolic processes might be altered by adjusting gut microbiota composition, which could increase efficacy and minimize adverse effects.

\section{B. Therapeutic Ketogenic Diet}

The ketogenic diet (KD) is a common and well-known, non-pharmacologic therapy used for many times in children with medically refractory epilepsy, according to recent research (Kossoff et al., 2018). It is a diet that's heavy in fat, high in protein, and low in carbohydrates. The fat-to-proteinto-carbohydrates-combined ratio in the diet is between 2:1 and $4: 1$. For every gram of protein and carbohydrate combined, the $4: 1$ ratio contains four times as much fat. Fat will provide $70-90 \%$ of the energy used in this condition. The conventional KD might alleviate epilepsy through a variety of mechanisms, including neurotransmitter regulation, brain energy metabolism, oxidative stress, and potassium channel modulation (Ulamek-Koziol et al., 2019). These explain another possible anticonvulsant mechanism of the ketogenic diet.

The ketogenic diet, in theory, causes ketosis, which is characterized by high blood levels of ketone bodies produced 
by the liver from dietary fat. In the body's cells, including the brain, ketones are utilized as an alternative source of energy for adenosin triphosphate (ATP) generation. Many biochemical, metabolic, and hormonal changes occur as a result of this metabolic shift, which may lead to lower neural excitability and fewer seizures (Neal et al., 2008). Furthermore, because GABA's most significant function in the brain is to reduce neuronal excitability, it plays a critical role in the onset and spread of seizure activity in the brain. The ketogenic diet has been shown to activate glutamic acid decarboxylase, which leads to GABA production. This diet has also been proven to affect GABA transaminase activity, which prevents GABA breakdown. The metabolic and temporary failure of GABAergic inhibition can be compensated for by enhancing energy metabolism with a ketogenic diet, but this will not prevent the development and spread of seizures. Thus, the GABAergic system is likely to be mediating another significant mechanism caused by the ketogenic diet in anticonvulsant action (Calderon et al., 2017).

The effectiveness of KD in medically refractory epilepsy in children was investigated by Neal et al., (2008) and Lambrects et al., (2017) in research. For the two experiments conducted, the responder rate, defined as patients who had a $50 \%$ reduction in the number of seizures, was 38 percent and 50 percent, respectively Furthermore, A 12-month KD based on olive oil might improve symptoms in 83.1 percent of patients with refractory epilepsy, equivalent to the results of a typical KD therapy, according to research by Guzel et al., (2019). The basic mechanisms of action of KD aren't completely understood, although current research has presented a few possibilities. Increased GABA-mediated inhibition, direct inhibitory activities of polyunsaturated fatty acids on ion channels, greater amounts of ATP, which is converted to the inhibitory mediator adenosine, increased mitochondrial biogenesis, and decreased oxidative stress are few of the examples (Rogawski et al., 2016). Recently, it has been suggested that the gut microbiota may play a role in the mechanism of action of KDs, which will be reviewed further.

\section{Probiotic as A Treatment}

Probiotics are living microorganisms that are advantageous to the host's health when given at the right dose (Hill et al., 2014). Bifidobacterium and Lactobacillus are two of the most prevalent probiotics (Yan et al., 2020). Prebiotics were described as a "substrate preferentially used by host microorganisms providing a health advantage" by the International Scientific Association for Probiotics and Prebiotics in 2020 (Swanson et al., 2020). Synbiotics are defined as "a mixture comprising live microorganisms and substrate(s) selectively utilized by host microorganisms that confers a health benefit on the host" and are divided into two types: synergistic synbiotics (the prebiotic is selectively used by the host microbes) and complementary synbiotics (each component appears to work separately) (Swanson et al., 2020). Probiotics have been shown in several studies to modify the gut microbiota and stabilize microbial ecosystems. Normalizing the composition of the gut microbiota may be advantageous on epilepsy. Probiotics have been shown to improve epileptic symptoms by boosting GABA levels in animals and Bifidobacteria and Lactobacillus levels in people (Peng et al., 2018; Gomez-Eguilaz et al., 2018).

According to Gomez et al., (2018) the frequency of seizures was decreased by $50 \%$ in $28.9 \%$ of drug-resistant epilepsy patients treated with a probiotic combination for four months, and $76.9 \%$ of these treated patients sustained a decreased seizure frequency four months after withdrawal. Adjuvant probiotics were found to lessen the frequency of seizures and might be utilized as an adjunct treatment to antiepileptic medication in this study (Gomez-Eguilaz et al., 2018). In the pentylenetetrazol (PTZ) mediated chemical kindling mouse model, on the other hand, Bagheri et al reported that the probiotic supplementation group did not reveal full kindling, and GABA elevated in mouse brain tissue, indicating that probiotic supplementation could significantly reduce seizure severity (Bagheri et al., 2019). Synbiotics or Lactobacillus fermentum MSK 408 might lessen the negative effects of KD without affecting its antiepileptic efficacy when treating PTZ-induced seizures in rats with KD (Eor et al., 2021). By modulating the gut microbiota, both KD and MSK 408 improve GABA metabolism (Eor et al., 2021). Antiepileptic properties are seen in several SCFAs, including propionate and butyrate. Total SCFAs were dramatically reduced after a month of intervention with the conventional KD, particularly acetate, propionate, and butyrate, which might be attributable to a reduction in the intake of fermentable carbs or a reduction in fermenting bacteria by KD (Ferraris et al., 2021). Synbiotics can help to enhance the gut microbiota, which is linked to SCFAs (Eor et al., 2021). By altering the gut microbiota, MSK 408 might affect SCFAs and recover serum lipid profile and tight junction protein mRNA expression in the gut and brain separately (Eor et al., 2021). These findings represent preliminary observations of supplemental probiotics in the therapy of drug-resistant epilepsy, and bigger placebocontrolled trials and more thorough animal research should be done to enhance theoretical validation and mechanism study. Probiotics might be potential as a supplementary therapy for refractory epilepsy, and it can be used together with KD treatments to decrease adverse effects (Calderon et al., 2017; Gomez-Eguilaz et al., 2018; Ferraris et al., 2021).

\section{CONCLUSION}

We noted in this review that the gut microbiota is becoming more well acknowledged as a significant component in epilepsy. Epilepsy is a complex condition that necessitates a knowledge of the connections between hereditary and environmental risk factors. Immunity and inflammation, metabolism, and peripheral and central neuronal signaling are all regulated by the gut microbiota, which are all connected to epileptogenesis in different ways. Different chemicals can alter the gut microbiota in a favorable or negative way, reducing or worsening epileptic episodes. Anti-epileptic medicines, the ketogenic diet, and probiotics are among them. Through their influence on the gut microbiota, these altering medicines may be regarded for improving epileptic symptoms. Therefore, deeper research into the microbiota's function in epilepsy might lead to the discovery of molecular foundations of epilepsy pathogenesis, biomarkers for illness and therapeutic responsiveness, and 
innovative therapies for refractory epilepsy.

\section{CONFLICT OF INTEREST}

Authors declare that they do not have any conflict of interest.

\section{REFERENCES}

Al-Ghezi, Z.Z., Busbee, P.B., Alghetaa, H., Nagarkatti, P.S., Nagarkatti, M. (2019). Combination of cannabinoids, delta-9- tetrahydrocannabinol (THC) and cannabidiol (CBD), mitigates experimental autoimmune encephalomyelitis (EAE) by altering the gut microbiome. Brain Behav Immun, 82, 25-35.

Borghi, E., \& Vignoli, A. (2019). Rett syndrome and other neurodevelopmental disorders share common changes in gut microbial community: a descriptive review. Int. J. Mol. Sci, 20, 17.

Blander, J.M., Longman, R.S., Iliev, I.D., Sonnenberg, G., Artis, D. (2017). Regulation of inflammation by microbiota interactions with the host. Nat Immunol, 18, 851-60.

Balosso, S., Liu, J., Bianchi, M.E., Vezzani, A. (2014). Disulfide-containing high mobility group box-1 promotes $\mathrm{N}$-methyl-D-aspartate receptor function and excitotoxicity by activating toll-like receptor 4- dependent signaling in hippocampal neurons. Antioxid Redox Signal, 21, 172640.

Bagheri, S., Heydari, A., Alinaghipour, A., Salami M. (2019). Effect of probiotic supplementation on seizure activity and cognitive performance in PTZ induced chemical kindling. Epilepsy Behav, 95, $43-50$.

Cryan, J.F., O’Riordan, K.J., Sandhu, K., Peterson, V., Dinan, T.G. (2020) The gut microbiome in neurological disorders. Lancet Neurol, 19, 17994.

Calderón, N., Betancourt, L., Hernández, L., Rada, P. (2017). A ketogenic diet modifies glutamate, gammaaminobutyric acid and agmatine levels in the hippocampus of rats: A microdialysis study. Neurosci. Lett, 642 , 158-62.

Drekonja, D., Reich, J., Gezahegn, S., Greer, N., Shaukat, A., MacDonald, R., et al. (2015). Fecal microbiota transplantation for clostridium difficile infection: a systematic review. Ann Intern Med, 162, 630-8.

de Theije, C.G.M., Wopereis, H., Ramadan, M., van Eijndthoven, T., Lambert. J., Knol, J., et al. (2014). Altered gut microbiota and activity in a murine model of autism spectrum disorders. Brain Behavior Immun, 37, 197-206.

Erny, D., Hrabě de Angelis, A.L., Jaitin, D., Wieghofer, P., Staszewski, O., David, E., et al. (2015). Host microbiota constantly control maturation and function of microglia in the CNS. Nat Neurosci, 18, 965-77.

Eor, J.Y, Tan, P.L, Son, Y.J, Kwak, M.J, Kim, S.H. (2021). Gut microbiota modulation by both lactobacillus fermentum MSK 408 and ketogenic diet in a murine model of pentylenetetrazole-induced acute seizure. Epilepsy Res, 169, 106506.

Fisher, R.S., Acevedo, C., Arzimanoglou, A., Bogacz, A., Cross, J.H., Elger, C.E., et al. (2014). ILAE official report: a practical clinical definition of epilepsy. Epilepsia, 55(4), 475-82.

Ferraris, C., Meroni, E., Casiraghi, M.C., Tagliabue, A., De Giorgis, V., Erba, D. (2021). One month of classic therapeutic ketogenic diet decreases short chain fatty acids production in epileptic patients. Front Nutr. 2021, 8, 613100 .

GBD 2016 Epilepsy Collaborators. (2019) Global, regional, and national burden of epilepsy, 1990-2016: A systematic analysis for the Global Burden of Disease Study. Lancet Neurol,18, 357-75.

Guzel, O., Uysal, U., Arslan, N. (2019). Efficacy and tolerability of olive oilbased ketogenic diet in children with drug-resistant epilepsy: a single center experience from turkey. Eur J Paediatr Neurol, 23, 143-51.

Gómez-Eguílaz, M., Ramón-Trapero, J.L., Pérez-Martínez, L., Blanco, J.R. (2018). The beneficial effect of probiotics as a supplementary treatment in drug-resistant epilepsy: A pilot study. Benef. Microbes, 9, 875-81.

He, Z., Cui, B.T., Zhang, T., Li, P., Long, C.Y., Ji, G.Z., et al. (2017). Fecal microbiota transplantation cured epilepsy in a case with Crohn's disease: The first report.World J Gastroenterol, 21, 23(19), 3565-8.

Hill, C., Guarner, F., Reid, G., Gibson, G.R., Merenstein, D.J., Pot, B., et al (2014). Expert consensus document. the international scientific association for probiotics and prebiotics consensus statement on the scope and appropriate use of the term probiotic. Nat Rev Gastroenterol Hepatol, 11, 506-14.

Jakobsson, H.E., Rodríguez-Piñeiro, A.M., Schütte, A., Ermund, A., Boysen, P., Bemark, M., et al. (2015). The composition of the gut microbiota shapes the colon mucus barrier. EMBO Rep, 16, 164-77.
Kossoff, E.H., Zupec-Kania, B.A., Auvin, S., Ballaban-Gil, K.R., Christina, B.A.G., Blackford, R., et al. (2018). Optimal clinical management of children receiving dietary therapies for epilepsy: updated recommendations of the international ketogenic diet study group. Epilepsia Open, 3(2), 175-92.

Lambrechts, D.A.J.E., de Kinderen, R.J.A., Vles, J.S.H., de Louw, A.J.A., Aldenkamp, A.P., Majoie, H.J.M. (2017). A randomized controlled trial of the ketogenic diet in refractory childhood epilepsy. Acta Neurol Scand, 135(2), 231-9.

Mendes, V., Galvão, I., Vieira, A.T. (2019). Mechanisms by which the gut microbiota influences cytokine production and modulates host inflammatory responses. J. Interf. Cytokine Res, 39, 393-409.

Mayer, E.A., Tillisch, K., Gupta, A. (2015). Gut/brain axis and the microbiota. J Clin Invest, 125(3), 926-38.

Maroso, M., Balosso, S., Ravizza, T., Liu, J., Aronica, E., Iyer, A.M, et al. (2010). Toll-like receptor 4 and high-mobility group box-1 are involved in ictogenesis and can be targeted to reduce seizures. Nat Med, $16,413-9$.

Maier, L., Pruteanu, M., Kuhn, M., Zeller, G., Telzerow, A., Anderson, E.E., et al. (2018). Extensive Impact of Non-Antibiotic Drugs on Human Gut Bacteria. Nature, 555, 623-8.

Neal, E.G., Chaffe, H., Schwartz, R.H., Lawson, M.S., Edwards, N., Fitzsimmons, G., et al. (2008). The ketogenic diet for the treatment of childhood epilepsy: a randomised controlled trial. Lancet Neurol, 7(6), 500-6.

O’Mahony, S.M., Clarke, G., Borre, Y.E., Dinan, T.G., Cryan, J.F. (2015) Serotonin, tryptophan metabolism and the brain-gut- microbiome axis. Behav Brain Res, 15(277), 32-48.

Peng, A., Qiu, X., Lai, W., Li, W., Zhang, L., Zhu, X., et al. (2018). Altered composition of the gut microbiome in patients with drug-resistant epilepsy. Epilepsy Res, 147, 102-7.

Rinninella, E., Raoul, P., Cintoni, M., Franceschi, F., Miggiano, G., Gasbarrini, A., et al. (2019). What is the healthy gut microbiota composition? A changing ecosystem across age, environment, diet, and diseases. Microorganisms, 7, 14.

Raymond, F., Ouameur, A.A., Déraspe, M., Iqbal, N., Gingras, H., Dridi, B., et al. (2016). The initial state of the human gut microbiome determines its reshaping by antibiotics. ISME J, 10, 707-20.

Rogawski, M.A., Löscher, W., Rho, J.M. (2016). Mechanisms of action of antiseizure drugs and the ketogenic diet. Cold Spring Harb Perspect Med, 2, 65.

Scheffer, I.E., Berkovic, S., Capovilla, G., Connolly, M.B., French, J., Guilhoto, L., et al. (2017). ILAE classification of the epilepsies: position paper of the ILAE commission for classification and terminology. Epilepsia, 58(4), 512-21.

Sgritta, M., Dooling, S.W., Buffington, S.A., Momin, E.N., Francis, M.B., Britton, R.A., et al. (2019). Mechanisms underlying microbialmediated changes in social behavior in mouse models of autism spectrum disorder. Neuron, 101, 246-59.

Stokes, J.M., Davis, J.H., Mangat, C.S., Williamson, J.R., Brown, E.D. (2014). Discovery of a small molecule that inhibits bacterial ribosome biogenesis. Elife, 3, e03574.

Swanson, K.S., Gibson, G.R., Hutkins, R., Reimer, R.A., Reid, G., Verbeke, K., et al. (2020). The international scientific association for probiotics and prebiotics (ISAPP) consensus statement on the definition and scope of synbiotics. Nat Rev Gastroenterol Hepatol, 17, 687-701.

Ułamek-Kozioł, M., Czuczwar, S.J., Januszewski, S., Pluta, R. (2019). Ketogenic diet and epilepsy. Nutrients, 11, 2510 .

van de Wouw, M., Boehme, M., Lyte, J.M., Wiley, N., Strain, C., O'Sullivan, O., et al. (2018). Short-chain fatty acids: microbial metabolites that alleviate stress-induced brain-gut axis alterations. J Physiol, 596, 4923-44.

Wang, H.X., \& Wang, Y.P. (2016). Gut microbiota-brain axis. Chin Med J (Engl), 129(19), 2373-80.

Warner, B.B. (2019). The contribution of the gut microbiome to neurodevelopment and neuropsychiatric disorders. Pediatr Res, 85(2), 216-24.

Yamashiro, Y. (2017). Gut microbiota in health and disease. Ann. Nutr. Metab, 71, 242-46.

Yan, F., \& Polk, D.B. (2020). Probiotics and probiotic-derived functional factors- mechanistic insights into applications for intestinal homeostasis. Front Immunol, 11, 1428.

Zocco, M.A., Ainora, M.E., Gasbarrini, G., Gasbarrini, A. (2007). Bacteroides thetaiotaomicron in the gut: molecular aspects of their interaction. Dig. Liver Dis, 39, 707-12.

Zimmermann, M., Zimmermann-Kogadeeva, M., Wegmann, R., Goodman, A.L. (2019). Separating host and microbiome contributions to drug pharmacokinetics and toxicity. Science, 363. 